\title{
Hacia la felicidad laboral: atender motivaciones y eliminar «temores digitales»
}

\section{Towards happiness in workplace: taking care of motivations and eliminating «digital fears»}

Dra. María-José Foncubierta-Rodríguez es profesora e investigadora de la Universidad de Cádiz (España) (mariajose.foncubierta@uca.es) (https://orcid.org/0000-0003-3231-5261)

Juan-Manuel Sánchez-Montero es investigador de la Universidad de Cádiz (España) (juanmanuel.sanchezmontero@alum.uca. es) (https://orcid.org/0000-0002-4940-0764)

\section{Resumen}

Considerando la motivación como integrante básico de la gestión de la felicidad en el trabajo, se analiza la valoración de determinados componentes de la misma en la era digital a partir del concepto de factor motivacional e higiénico de Herzberg. Se comprobará si existe alguna asociación entre el perfil sociodemográfico del trabajador y ciertos factores a «higienizar». Se realiza un cuestionario en muestra de empresas de la Bahía de Algeciras, en la provincia de Cádiz (España), en el Estrecho de Gibraltar, zona ésta que resulta de especial relevancia estratégica al ser ruta de tránsito mundial principal de personas y de mercancías, y punto de encuentro de dos continentes, dos mares y dos culturas. Mediante cuestionario validado, se recogen las opiniones de trabajadores sobre los factores establecidos como motivadores y desmotivadores (temores). Los datos, depurados, son tratados con el programa SPSS.25. Se confirma que los factores motivacionales propuestos son altamente valorados. La falsedad de datos, el miedo a ser sustituidos en las tareas y la «despersonalización» del trabajo son las cuestiones más temidas, cuya ausencia hay que procurar. Se percibe también, asociación de determinadas variables sociodemográficas del trabajador con el grado en que se manifiestan alguno de esos temores. La originalidad principal de este trabajo estriba en que se aportan y analizan factores motivacionales e higiénicos de nueva aparición en lo laboral, que, como tales, habrán de ser gestionados.

\begin{abstract}
Motivation is a basic element of the 'Happiness Management' model at work. Its components are analyzed in the digital era under the Herzberg Theory of the motivational and hygienic factors, to verify if an association between worker sociodemographic profile and these factors is detected, which would allow the managers to «sanitize» them. In a group of companies in Algeciras Bay, province of Cádiz (Spain), in the Strait of Gibraltar, a survey has been done. This area is of special strategic relevance, being the main transit route for people and merchandise worldwide, and a meeting point for two continents, two seas and two cultures. By a validated questionnaire, the opinions of workers are collected about the issues that have been established as motivating and demotivating factors (fears). Refined data are treated with SPSS.25 program. It is confirmed that the motivational factors are highly valued. Falsity of the data, fear of being substituted at job, and depersonalization because automation are the most feared topics. An association of worker sociodemographic variables and the level to which some of these fears are manifested is found out. The main originality of this paper lies in the contribution and analysis of new factors, motivational and hygienic, of digital age in workplace, which must be managed.
\end{abstract}

\section{Palabras clave I keywords}

Motivación, robotización laboral, Herzberg, gestión de la felicidad, satisfacción laboral, teorías de la motivación, temores en la era digital, empresa

Motivation, robotization of jobs, Herzberg, happiness management, job satisfaction, motivation theories, fears at digital era, company.

Cómo citar: Foncubierta-Rodríguez, M.J. y Sánchez-Montero, J.M. (2019). Hacia la felicidad laboral: Atender motivaciones y eliminar «temores digitales». Retos Revista de Ciencias de la Administración y Economía, 9(18), 239-257. https://doi.org/10.17163/ret.n18.2019.04 


\section{Introducción}

\subsection{Estado de la cuestión}

Con la llegada del nuevo siglo, y, sobre todo, de la actual década, un concepto que parecía incluso ajeno al contexto laboral ha ido tomando fuerza a través de los estudios realizados por expertos: el de la felicidad en el trabajo. Ya sea tratando directamente el «Happiness Management» (González-Díaz, 2018), como determinadas teorías, derivadas o complementarias de la misma, como «Happy-Performing Managers» (v. gr. Hosie et al., 2019), la literatura muestra que, tanto para empleados como para directivos, la conclusión es similar: personas más felices, con mayor bienestar emocional en el entorno de trabajo, muestran una mayor motivación para la realización del mismo; y ello acontece en empresas, pero también en organizaciones sin ánimo de lucro (Bashir et al., 2019).

Felicidad, satisfacción y motivación son términos distintos, pero claramente interrelacionados. La Real Academia de la Lengua Española (RAE) define la motivación como el estímulo o interés que determina en parte las acciones de una persona. $\mathrm{Y}$, así, que el empleado se encuentre motivado a realizar su trabajo y a lograr los objetivos fijados, podría considerarse tanto una finalidad (Price \& Reichert, 2017; Taipale et al., 2011; Baptiste, 2007), como un instrumento (Gaitán et al., 2015; Meyers et al., 2013; Zelenski et al., 2008, Chalofsky, 2003 en Arslan \& Roudaki, 2019) del «Happiness Management».

Los estudios que en la última década han relacionado la gerencia de la felicidad en el trabajo con el rendimiento o la productividad (tesis del «Happy Productive Worker» de Wright \& Staw, 1999'1 , analizada ampliamente por Ledford, 1999²), sostienen una asociación, en determinadas condiciones, entre empleados más felices y un mejor resultado laboral (Moccia, 2016; López \& Fierro, 2015; Fisher, 2010). Ello es especialmente importante en trabajos complejos que necesitan de soluciones creativas (Kang et al., 2016), lo que podría aplicarse al actual entorno digital.

En esta relación interviene también la motivación. Cropanzano y Wright (2001) concluyen que hay una alta asociación entre el estado de depresión, o poca felicidad, en el trabajo del empleado y la baja energía y motivación que muestre al realizarlo, llevándole a un escaso rendimiento. El nivel de desempeño laboral es el resultado de los comportamientos de las personas en el contexto social y motivacional en el que se realizan el trabajo organizacional (van Scotter \& Motowidlo, 1996, en Hosie et al., 2019, p.10; Ravina et al., 2017). La relación es compleja y multivariante. Zelenski et al. (op. cit.) argumentan que en esa función han de entrar a valorarse emociones, personalidades, tareas, y motivaciones, entre otros factores.

El presente estudio parte de un modelo en el que la motivación es instrumento para el logro de la felicidad en el entorno laboral (figura 1). Se realiza un análisis

1 Wright, T. A., \& Staw, B. M. (1999). Affect and favorable work outcomes: Two longitudinal tests of the happy-productive worker thesis. Journal of Organizational Behavior, 20. https://doi.org/10.1037/10614087.53.3.182.

2 Ledford, G.E. (1999). Happiness and Productivity revisited. Journal of Organizational Behavior, 20. https://doi. org/10.1002/(SICI)1099-1379(199901)20:1<25::AID-JOB913>3.0.CO;2-K. 
exploratorio para detectar motivaciones y desmotivaciones en el actual contexto digital laboral, que oriente a la gerencia sobre a qué cuestiones prestar más atención de cara a lograr un paso más hacia la «Happy Company». Y ello, porque es papel fundamental de la gerencia mantener el equilibrio afectivo/emocional de sus equipos, detectando las inconformidades que puedan darse, las desmotivaciones, para procurar solventarlas o limarlas en lo posible (Goleman, 1995, en Romero-Rodríguez et al., 2019 , p. 25) Y porque, como sostiene la RAE en una de las acepciones del término, la felicidad es la ausencia de tropiezos e inconvenientes.

Figura 1. Papel de la motivación en el modelo del «Happiness Management»

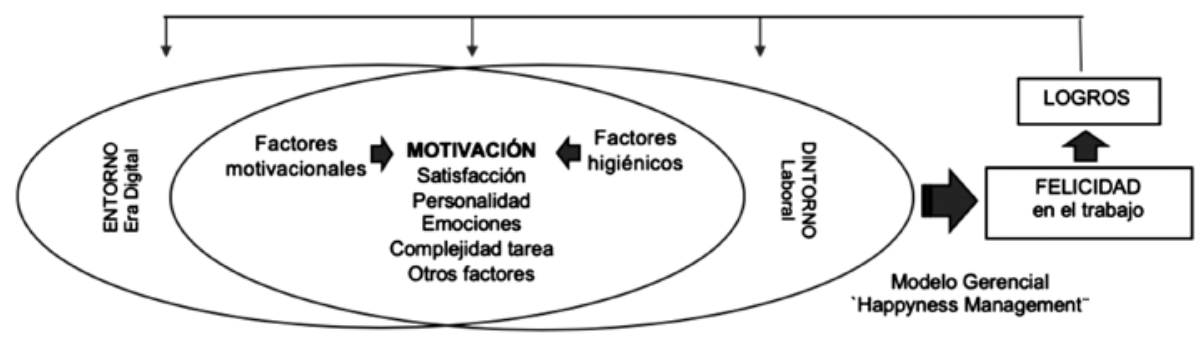

\subsection{La motivación laboral en la era digital y la Teoría de Herzberg}

Diversas son las definiciones que en la literatura describen a la motivación. En sentido amplio, ésta se trata de un estado emocional que se genera en una persona como consecuencia de la influencia en su comportamiento de determinados factores (Koenes, 1996). En el ámbito empresarial, hace referencia al proceso que explica la intensidad, dirección y persistencia del esfuerzo de un individuo para alcanzar una meta, siendo la intensidad el esfuerzo puesto en la realización de la tarea, la dirección, hacia dónde apunta ese esfuerzo, a qué logro o meta, y la persistencia, la duración en que se mantiene el esfuerzo (Robbins \& Judge, 2009).

Contribuye al grado de compromiso de la persona; es un proceso que ocasiona, activa, orienta, dinamiza y mantiene el comportamiento de los individuos hacia la realización de objetivos esperados (López, 2005, p.26).

\section{Tabla 1. Nuevos factores motivacionales en la era digital}

\begin{tabular}{|c|c|}
\hline Cuestión & Nombre de la variable \\
\hline \multicolumn{2}{|l|}{ Sistemas de información } \\
\hline $\begin{array}{l}\text { La digitalización y automatización de los puestos de trabajo au- } \\
\text { menta la motivación de los empleados. }\end{array}$ & MOTIVAC \\
\hline Contactar a través de redes amplía mi mundo como trabajador/a. & REDES \\
\hline $\begin{array}{l}\text { Disponer de información a través de Cloud Computing (Nube), o } \\
\text { Big Data, facilita mi trabajo. }\end{array}$ & BIGDATA \\
\hline
\end{tabular}




\section{Sistemas de información}

En el marco actual; ante la cuarta revolución industrial (Industria 4.0), la realidad "M2M" (machine to machine), es decir la transmisión y gestión de información entre máquinas, será positiva para un más eficiente desarrollo del trabajo.

En general, usar las nuevas tecnologías digitales en mi trabajo me resulta motivador.

Considero que una política de formación/actualización digital continua para los empleados es muy motivadora en la era actual.

M2M

Contextos de trabajo y relaciones

Trabajar con compañeros de distintos grados de cultura digital (babby boomers, generación $\mathrm{X}$, generación $\mathrm{Z}$ o millennials...) resulta motivador.

Me gusta el que las nuevas tecnologías me permitan trabajar colaborativamente con otras personas.

Trabajar en espacios físicos amplios, diáfanos y abiertos, que fomenten la interrelación y la transparencia entre los compañeros, y no en espacios estancos y separados, resulta motivador.

Tener la posibilidad de trabajar desde espacios físicos distintos a los de mi puesto de trabajo o empresa (teletrabajo) resulta motivador.

Me motiva trabajar en la diversidad, lo que en la nueva era se llaman "contextos de hibridación", es decir, con personas de distintas áreas de conocimiento, de distintas culturas, con distintos puntos de vista.

Me motiva el hecho de que las organizaciones tiendan a la "holocracia", es decir, a poder prescindir de cargos o directivos, dada la preparación y autonomía de los trabajadores, y su vinculación en equipos multidisciplinares, o híbridos, que trabajen colaborativamente.

El presente trabajo toma como base de referencia la «Teoría de la Motivación» de Herzberg et al. (1959). Son varias las investigaciones que han utilizado esta teoría como marco hasta nuestros días, en múltiples sectores o ámbitos profesionales (p.e., en Salud: Albohoseini et al., 2018, Petersen et al., 2017, Torkaman et al., 2017; en Educación: Verma \& Sharma, 2018, Emiroglu et al., 2017, Escardibul \& Afcha, 2017, Rizkallah \& Seitz, 2017; en el Sector Público: Khoshnevis \& Tahmasebi; en Hostelería: Cech et al., 2015), y en culturas diversas (Warrier \& Prasad, 2018; Rahman et al., 2017). No obstante, son escasas aún las dedicadas al entorno laboral en la era de la digitalización. Miraz et al. (2016) realizan un análisis de rasgos en las websites de determinadas organizaciones, tomando el enfoque de la «Teoría de los Factores Motivacionales e Higiénicos» de Herzberg. Con el presente estudio se pretende contribuir, aun con sus limitaciones, a cubrir este gap en la literatura. 
Figura 2. Factores «digitales» en la Teoría de la Motivación de Herzberg
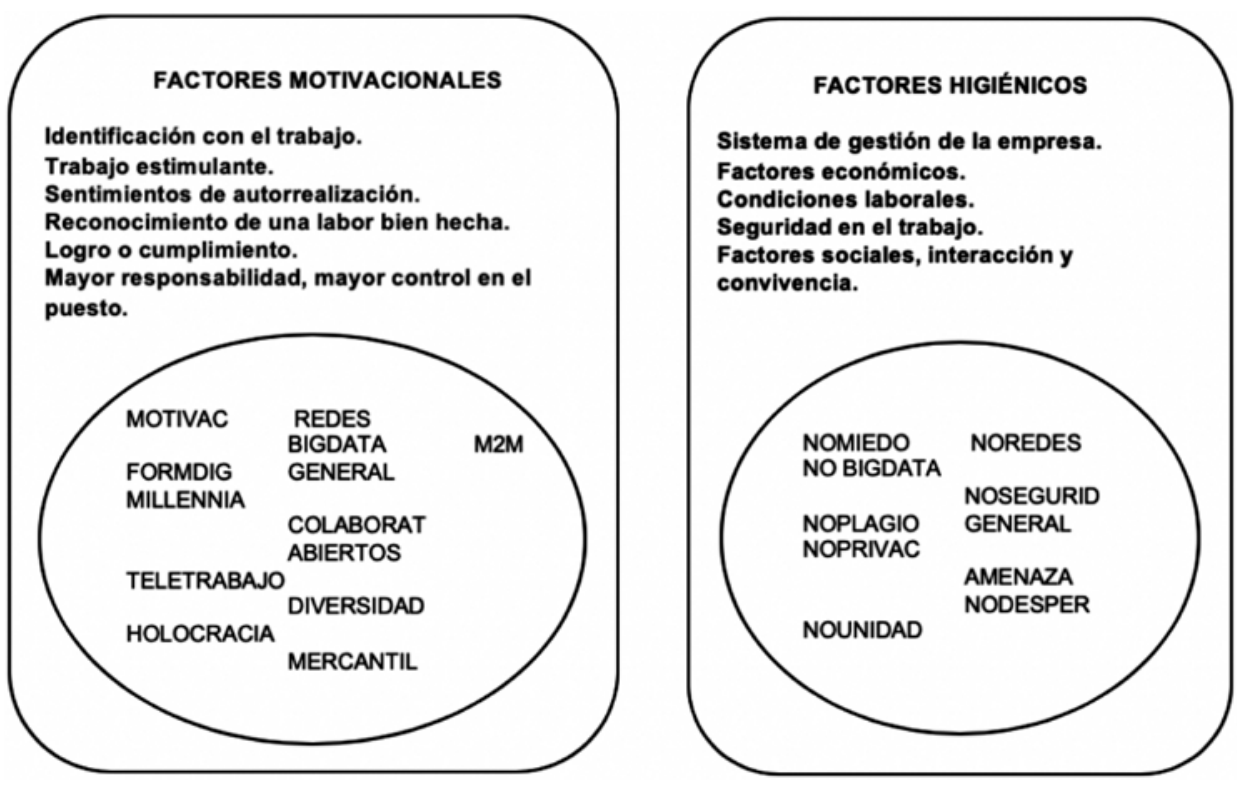

Herzberg habla de dos tipos de factores: aquéllos que crean motivación (motivacionales), y aquéllos cuya ausencia genera desmotivación (higiénicos). Entre los expertos, los términos motivación y satisfacción se mezclan a la hora de enunciar las bases de esta teoría (Khoshnevis \& Tahmasebi, 2015). El efecto para la organización es que si ésta elimina los factores que crean insatisfacción, es decir, aporta únicamente factores higiénicos, ello no asegurará la satisfacción de sus trabajadores (Ghahremani et al., 2014).

La originalidad de este trabajo radica en que se añaden a los dos grupos de factores tradicionales, tanto motivacionales como higiénicos, algunos de nueva aparición, característicos de la era digital, y que, ineludiblemente, forman, o van a ir formando progresivamente, parte de las condiciones habituales del contexto laboral (figura 2).

Las tablas 1 y 2 muestran los ítems en que se han concretado los citados factores. Aunque se trata de un grupo limitado, se han debatido y perfilado con expertos consultados durante el diseño del cuestionario utilizado, en la fase de pretest, como aquéllos que se experimentan con mayor frecuencia en el nuevo entorno laboral. 


\section{Tabla 2. Nuevos temores a eliminar por aporte de factores higiénicos en la era digital}

\begin{tabular}{|l|c|}
\hline \multicolumn{1}{|c|}{ Cuestión } & $\begin{array}{l}\text { Nombre de } \\
\text { la variable }\end{array}$ \\
\hline $\begin{array}{l}\text { La digitalización y automatización produce desmotivación sobre los empleados, } \\
\text { por el miedo de éstos a ser sustituidos por sistemas informáticos y/o máquinas. }\end{array}$ & NOMIEDO \\
\hline $\begin{array}{l}\text { Considero negativo el contacto a través de redes pues no permite la cercanía } \\
\text { personal. }\end{array}$ & NOREDES \\
\hline $\begin{array}{l}\text { Disponer de información a través de Cloud Computing (Nube), o Big Data, me } \\
\text { causa el temor de estar trabajando con datos poco analizados, e incluso falsos. }\end{array}$ & NOBIGDATA \\
\hline $\begin{array}{l}\text { En la actualidad el intenso uso de redes para el trabajo me despierta el temor } \\
\text { de que se produzcan fallos de seguridad (ciberseguridad): hackers, bots, per- } \\
\text { files falsos, etc. }\end{array}$ & NOSEGURID \\
\hline $\begin{array}{l}\text { En la actualidad, la facilidad de acceso a la información a través de las redes } \\
\text { me despierta el temor de que puedan plagiar los resultados de mi trabajo. }\end{array}$ & NOPLAGIO \\
\hline $\begin{array}{l}\text { La continua conexión digital de mi empresa, a través de redes, me despierta el } \\
\text { temor a la pérdida de privacidad. }\end{array}$ & NOPRIVAC \\
\hline $\begin{array}{l}\text { En la nueva era digital, la progresiva automatización/robotización resulta una } \\
\text { amenaza para mantener mi puesto de trabajo. }\end{array}$ & AMENAZA \\
\hline $\begin{array}{l}\text { En la nueva era digital, la progresiva automatización/robotización despersonal- } \\
\text { izará, y empobrecerá, por tanto, el trabajo. }\end{array}$ & NODESPER \\
\hline $\begin{array}{l}\text { Mercantilizar el trabajo es una forma más de romper la unidad, y, por tanto, } \\
\text { el poder, de los trabajadores como colectivo. }\end{array}$ & NOUNIDAD \\
\hline
\end{tabular}

\section{Materiales y método}

La investigación se centra en el tejido empresarial del arco de la Bahía de Algeciras, zona de negocio internacional que alberga al segundo polo industrial español y el primer puerto del Mediterráneo en volumen de mercancías; fisionomía económica que obliga a una continua digitalización de los negocios principales y derivados, con sus ventajas e inconvenientes.

Se selecciona, de entre el tejido empresarial, una muestra de 180 entidades, por muestreo aleatorio estratificado. A cada empresa se le remite una carta de presentación del estudio, solicitándole su colaboración y garantizando el anonimato de los datos y su uso exclusivo para la investigación. La carta es dirigida a la persona responsable del departamento o área de Recursos Humanos, o afín, en el convencimiento de que será el área más sensible a la materia que se trata. De ellas, y tras un proceso de depuración, queda una muestra final de 114 elementos.

Se trata de un estudio exploratorio, basado en metodología cuantitativa. El instrumento para la recopilación de las percepciones de los trabajadores es un cuestionario diseñado ad hoc, pues, aunque la literatura ofrece diversos modelos para medir la motivación, el hecho de ubicarlo en un contexto digital supone una novedad. El cuestionario es previamente validado, en primer lugar, mediante pretest por exper- 
tos en Recursos Humanos, que asistieron en la depuración y el complemento de su redacción para una mayor consistencia e inteligibilidad, y, en segundo lugar, por la prueba de fiabilidad estadística Alfa de Cronbach, que resulta de 0.896, quedando validado, según acuerdo de la literatura, al ser superior a 0.7 (Corbetta, 2010).

A la persona a la que se dirige la carta se le solicita que traslade la misma y el propio cuestionario a dos empleados de su organización, sin poner condiciones en cuanto a edad, sexo, antigüedad, puesto, formación, departamento..., procurando no sesgar la muestra.

En el cuestionario las opiniones se reflejan a través de una escala de Likert, siendo 1 el valor mínimo posible, que implica "Muy en desacuerdo», y 5 el valor máximo, que supone un "Muy de acuerdo». Los datos son tratados con el software estadístico IBM SPSS.25.

Al participante se le plantea una serie de preguntas para definir su perfil sociodemográfico. Se tratan de variables cualitativas nominales, ya sean de carácter binomial (sexo) o multinomiales (edad, sector y tamaño). Para operar en el análisis de los mismos, se renombran las categorías, y, dado que la muestra apenas supera el centenar de casos, para algunas variables se agregan categorías a fin de que contemplen un mayor número de elementos. En la tabla 3 ( $1^{\text {a }}$ transformación) quedan indicadas dichas modificaciones.

Tabla 3. Codificación de las variables sociodemográficas

\begin{tabular}{|c|c|c|}
\hline & $\begin{array}{c}\text { Variables de perfil } \\
\left.\text { sociodemográfico ( } 1^{\text {a }} \text { transformación }\right)\end{array}$ & $\begin{array}{c}\text { Variables de perfil } \\
\text { sociodemográfico } \\
\left(2^{\circ} \text { transformación: binarias }\right)\end{array}$ \\
\hline Variable & Categorías & Categorías \\
\hline Sexo & \multicolumn{2}{|l|}{$\begin{array}{l}\text { 0. Mujer: } 46 \\
\text { 1. Hombre: } 68\end{array}$} \\
\hline Edad & \multicolumn{2}{|c|}{$\begin{array}{l}\text { 0. NO JOVEN (más de } 35 \text { años): } 65 \\
\text { 1. JOVEN (hasta } 35 \text { años): } 49\end{array}$} \\
\hline Sector & $\begin{array}{l}\text { 1. EDUCA: } 31 \\
\text { (Educación) } \\
\text { 2. TPTE: } 14 \\
\text { (Automoción,transporte) } \\
\text { 3. SECUND: } 23 \\
\text { (Industria y minería+Químico, farmaceútico, } \\
\text { sanitario+Construcción) } \\
\text { 4. SERVICIO: } 31 \\
\text { (Servicios a empresas+Finanzas+Servicios recrea- } \\
\text { tivos, culturales, ocio) } \\
\text { 5. COMHOST: } 15 \\
\text { (Comercio+Alimentación, hostelería, restauración) }\end{array}$ & $\begin{array}{l}\text { 0. EDUCA+TPTE } \\
\text { 1. Resto (SECUND+ } \\
\text { SERVICIO+COMHOST) }\end{array}$ \\
\hline Tamaño & $\begin{array}{l}\text { 1. MICMIN (hasta } 49 \text { trabajadores): } 43 \\
\text { 2. PYME (de } 50 \text { a } 249 \text { trabajadores): } 28 \\
\text { 3. GRANDE (de } 250 \text { o más trabajadores): } 43\end{array}$ & $\begin{array}{l}\text { 0. GRANDE } \\
\text { 1. MICMIN+PYME }\end{array}$ \\
\hline
\end{tabular}




\section{Resultados}

\subsection{Primeros resultados}

Todos los factores motivacionales propuestos consiguen puntuaciones medias por encima de 3, esto es, son aceptados como tales. Claramente destacan las motivaciones originadas por trabajar en «contextos de hibridación», con personas de distintas áreas de conocimiento, de distintas culturas, con distintos puntos de vista, y por disfrutar de una política formativa continua de actualización digital que la empresa plantee para sus empleados. Sus respectivas desviaciones típicas son las menores de todo el listado (tabla 4).

\section{Tabla 4. Principales descriptivos de los factores}

\begin{tabular}{|c|c|c|c|c|c|c|c|c|c|}
\hline & 宜 & 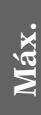 & 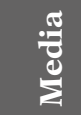 & $\stackrel{8}{8}$ & & $\dot{B}$ & 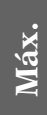 & 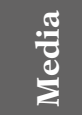 & 㝴 \\
\hline Motivac & 1 & 5 & 3.202 & 0.833 & Nomiedo & 2 & 5 & 3.246 & 1.052 \\
\hline Redes & 2 & 5 & 4.009 & 0.804 & Noredes & 1 & 5 & 3.272 & 1.099 \\
\hline Bigdata & 2 & 5 & 3.904 & 0.902 & Nobigdata & 1 & 5 & 3.465 & 1.138 \\
\hline M2m & 2 & 5 & 3.570 & 0.704 & Nosegurid & 1 & 5 & 2.763 & 1.292 \\
\hline General & 2 & 5 & 4.123 & 0.742 & Noplagio & 1 & 5 & 2.921 & 1.256 \\
\hline Formdig & 3 & 5 & 4,246 & 0.659 & Noprivac & 1 & 5 & 2.526 & 1.146 \\
\hline Millennia & 1 & 5 & 3.921 & 0.864 & Amenaza & 1 & 5 & 2.807 & 1.233 \\
\hline Colaborat & 2 & 5 & 4.070 & 0.725 & Nodesper & 1 & 5 & 3.105 & 1.100 \\
\hline Abiertos & 1 & 5 & 3.921 & 1.090 & Nounidad & 1 & 5 & 2.570 & 0.902 \\
\hline Teletrabajo & 2 & 5 & 3.895 & 0.813 & & & & & \\
\hline Diversidad & 3 & 5 & 4.281 & 0.698 & & & & & \\
\hline Holocracia & 1 & 5 & 3.202 & 1.099 & & & & & \\
\hline
\end{tabular}

Los calificados como temores a «higienizar» presentan una mayor irregularidad. La posibilidad de trabajar con datos falsos, la falta de cercanía personal que conlleva el comunicarse a través de redes, el miedo a ser sustituidos por sistemas informáticos y máquinas y la despersonalización que puede acarrear la progresiva automatización de las tareas son los más temidos. Los factores relacionados con la falta de seguridad, el plagio, la no privacidad de la información particular, el sentir amenazado el empleo por la progresiva automatización y el riesgo de que la mercantilización de las tareas conlleve la pérdida de unidad del colectivo de trabajadores, muestran medias por debajo de 3. No obstante, un factor común en todos ellos es 
que también son elevadas sus desviaciones típicas, algunos participantes no los considera en absoluto como cuestión a temer, otorgándoles un 1, mientras que otros les conceden el mayor valor, 5.

Cabe, pues, plantearse, y en ello se centra el presente trabajo, si se dan comportamientos distintos entre las distintas categorías de las diferentes variables sociodemográficas, a las que se ha denominado variables de perfil. Esto es, si es posible asociar los temores "digitales» (variables dependientes, en su caso) a determinados género o edad, o a pertenecer a sectores económicos, o a colectivos de tamaños de empresas, donde éstos se puedan experimentar en mayor medida (variables independientes, o de control).

\subsection{Estudio de asociaciones}

En un primer paso se analizan las medias de las diferentes categorías de cada una de las variables de perfil (figura 3). Se observa que en las categorías de sexo las medias de los diversos factores son similares, prácticamente los perfiles gráficos se superponen. Para las dos edades codificadas, jóvenes y no jóvenes, parece vislumbrarse una clara diferencia en AMENAZA, más percibida por los jóvenes. También se puede comprobar en NOSEGURID, aunque más levemente. La diversidad es mayor en cuanto a las categorías de la variable Sector, donde cabe sospechar de diferencias en NOBIGDATA, NOSEGURID, NOPLAGIO, NOPRIVAC, AMENAZA y NOUNIDAD. Transporte y Educación parecen ser los sectores donde menores son dichos miedos; el sector Secundario y el de Servicios donde más. Con respecto al tamaño de la empresa, las de menos de 50 empleados son donde mayor es el temor medio a NODESPER, y de forma menos evidente en NOMIEDO y NOPRIVAC, junto con las PYME.

Figura 3. Comparación de medias de los factores higiénicos
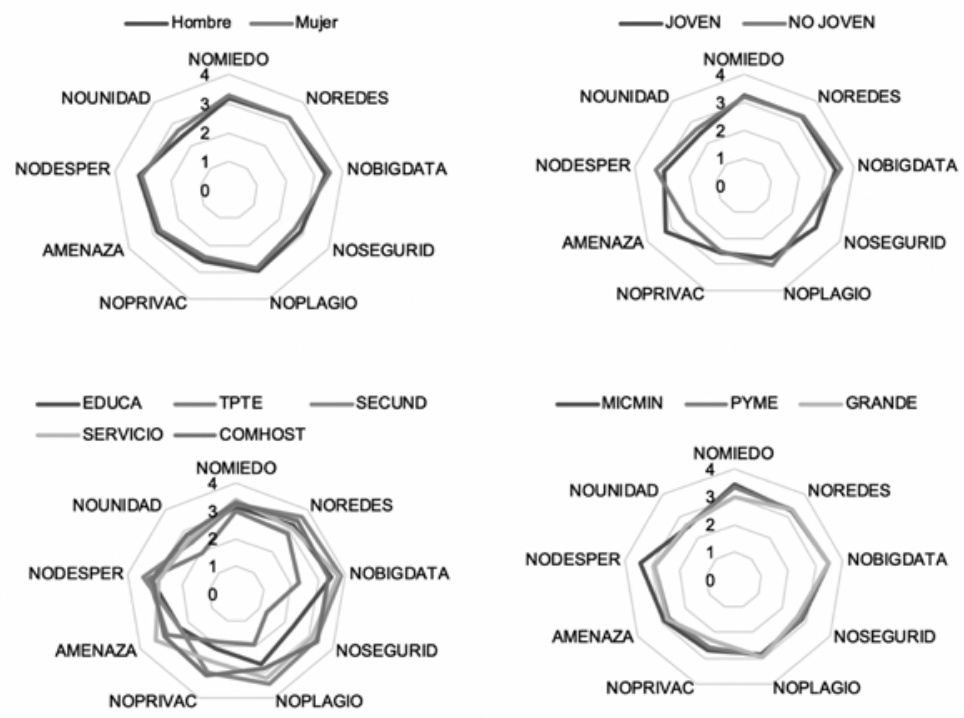
Comprobando las respectivas tablas de contingencia, se agrupan las categorías de las variables Sector y Tamaño para convertir a éstas en variables binarias. El proceso, lógicamente, se hace uniendo en un mismo grupo las categorías que han demostrado tener una distribución similar (tabla 3, 2a transformación). A partir de esta transformación se realizan las pruebas de normalidad como elemento de partida de las medidas de asociación a utilizar. Para todas las distribuciones bicategóricas de las variables de perfil las pruebas de Kolmogorov-Smirnov y de Shapiro-Wilk, muestran que dichas distribuciones no son normales (sig.<0,05).

De los resultados obtenidos en el cálculo de las medias de las variables a «higienizar» para las distintas categorías se establecen las sucesivas hipótesis nulas, y sus respectivas hipótesis complementarias:

\section{Sexo:}

H01n: no se da asociación entre la variable Sexo y la variable NOMIEDO. Las medias de las distribuciones de sus categorías son iguales.

H1 1n: se da asociación entre la variable Sexo y la variable NOMIEDO. Las medias de las distribuciones de sus categorías no son iguales.

Se plantean similares pares de hipótesis con:

Edad: H02 y H12 (AMENAZA); H03 y H13 (NOSEGURID).

\section{Sector:}

H04 y H14 (NOBIGDATA); H05 y H15 (NOSEGURID); H06 y H16 (NOPLAGIO); H07 y H17 (NOPRIVAC), H08 y H18 (AMENAZA); H09 y H19 (NOUNIDAD).

\section{Tamaño:}

H010 y H110 (NODESPER); H011 y H111 (NOMIEDO); H012 y H112 (NOPRIVAC).

Para el contraste de estos pares de hipótesis obtenidos, se procede a realizar la prueba no paramétrica U de Mann-Whitney, que lleva a rechazar algunas de las hipótesis nulas, indicando asociación. Ello, junto a las tablas de contingencia, la prueba de Chi-cuadrado para el cálculo del coeficiente de correlación ( $\rho$ ) y la estimación del riesgo $(\mathrm{RR})$ conducen a unas primeras interpretaciones:

Sexo:

H11.3: se da asociación con la variable NOBIGDATA.

Chi-Cuadrado: confirma la significación estadística (Sig.<0,05): 0,042

Interpretación (RR): un hombre teme 2,952 veces más esta cuestión que una mujer.

H11.8: asociación con NODESPER.

Chi-Cuadrado:0,004

Interpretación: una mujer teme 3,241 veces más esta cuestión que un hombre. 


\section{Edad:}

H12: asociación con AMENAZA.

Chi-Cuadrado:0,008

Interpretación: una persona joven (hasta 35 años) teme 2,968 veces más esta cuestión que una persona no joven.

Sector:

Aparece asociación con NOREDES.

Chi-Cuadrado: 0,008

Interpretación: una persona que trabaja en sectores que no sean los de Educación y de Automoción y transporte teme 2,879 veces más esta cuestión que una persona que trabaja en estos sectores.

H15: asociación con NOSEGURID.

Chi-Cuadrado: 0,000

Interpretación: una persona que trabaja en los sectores de Educación y de Automoción y transporte teme 5,882 veces más esta cuestión que una persona que no trabaja en estos sectores.

H16: asociación con NOPLAGIO.

Chi-Cuadrado: 0,002

Interpretación: una persona que trabaja en los sectores de Educación y de Automoción y transporte teme 3,428 veces más esta cuestión que una persona que no trabaja en estos sectores.

H17: asociación con NOPRIVAC.

Chi-Cuadrado: 0,014

Interpretación: una persona que trabaja en los sectores de Educación y de Automoción y transporte teme 2,684 veces más esta cuestión que una persona que no trabaja en estos sectores.

\section{Tamaño:}

H111: asociación con NOMIEDO.

Chi-Cuadrado: 0,009

Interpretación: una persona que trabaja en micro empresas o en pequeñas y medianas empresas teme 3,032 veces más esta cuestión que una persona que trabaja en una gran empresa.

H112: asociación con NOPRIVAC.

Chi-Cuadrado: 0,032

Interpretación: una persona que trabaja en una gran empresa teme 2,374 veces más esta cuestión que una persona que lo hace en micro empresas o en pequeñas y medianas empresas.

El número de participantes es un poco mayor que 100 en la muestra total, no así en las submuestras por categorías. No obstante, se adopta la decisión de realizar el contraste de hipótesis como si las distribuciones gozasen de normalidad, a través 
de la prueba t-Student de comparación de medias entre las distribuciones de cada variable de perfil. Los resultados son coincidentes para las variables Sexo, Edad y Sector, con $\rho$ iguales o similares, que confirman la significación estadística. Para la variable Tamaño sólo se da coincidencia, esto es, la prueba sólo señala asociación, para la variable NOPRIVAC.

Se ha utilizado el riesgo, RR, como medida de cuánto más teme una de las categorías sobre la otra. Sin embargo, cabe profundizar en otro tipo de estudios, para confirmar o no, estas primeras conclusiones. Para ello, se recurre a medir la regresión logística binaria de cada variable dependiente según las primeras conclusiones para cada categoría de las variables de perfil o de control. Éstas han pasado, previamente, a «nominalizarse», tomando los valores 4 y 5 como «Sí teme», y el resto, 1, 2 y 3, como «No teme».

Tabla 5. Resumen de medidas de asociación encontradas

\begin{tabular}{|c|c|c|c|c|c|c|c|}
\hline \multirow{2}{*}{$\begin{array}{c}\text { Variable } \\
\text { Perfil }\end{array}$} & \multirow{2}{*}{$\begin{array}{c}\text { Variable } \\
\text { dependiente }\end{array}$} & \multirow{2}{*}{$\mathbf{r}$} & \multirow{2}{*}{$\mathbf{O R}$} & \multicolumn{2}{|c|}{ IC95\% de la OR } & \multicolumn{2}{|c|}{ Pronosticado } \\
\hline & & & & Inferior & Superior & 0 & 1 \\
\hline \multirow{2}{*}{ Sexo } & Nobigdata & .048 & .339 & .116 & .991 & $100 \%$ & $0.00 \%$ \\
\hline & Nodesper & .005 & 3.239 & 1.435 & 7.313 & $100 \%$ & $0.00 \%$ \\
\hline Edad & Amenaza & .009 & .337 & .149 & .762 & $100 \%$ & $0.00 \%$ \\
\hline \multirow{5}{*}{$\begin{array}{l}\text { Sector } \\
\text { (Recodificada) }\end{array}$} & Noredes & .009 & .347 & .158 & .766 & $50.8 \%$ & $73.6 \%$ \\
\hline & \multirow{2}{*}{ Nosegurid } & \multirow[b]{2}{*}{.000} & \multirow[b]{2}{*}{5.875} & \multirow[b]{2}{*}{2.556} & \multirow[b]{2}{*}{13.505} & $79.7 \%$ & $60.0 \%$ \\
\hline & & & & & & \multicolumn{2}{|c|}{ Total: $70.2 \%$} \\
\hline & Noplagio & .002 & 3.429 & 1.561 & 7.528 & $72.7 \%$ & $56.3 \%$ \\
\hline & Noprivac & .015 & 2.685 & 1.208 & 5.971 & $73.5 \%$ & $42.9 \%$ \\
\hline \multirow{2}{*}{$\begin{array}{l}\text { Tamaño } \\
\text { (Recodificada) }\end{array}$} & Nomiedo & .010 & .330 & .141 & .769 & $100 \%$ & $0.00 \%$ \\
\hline & Noprivac & .034 & 2.374 & 1.067 & 5.283 & $73.5 \%$ & $46.2 \%$ \\
\hline
\end{tabular}

En lugar de las RR, en las regresiones logísticas se muestran las OR, esto es, la relación entre la oportunidad de que se dé el evento en el subgrupo codificado como 1 para cada variable de control, es decir, de que sus integrantes sientan temor a la circunstancia estudiada, frente a la oportunidad de que no se dé. Los coeficientes de correlación, r, las OR, los valores inferiores y superiores, así como el porcentaje de valores pronosticados con éxito (que coincidan con los reales) en el caso de integrar esa variable en un modelo de dependencia, o asociación, quedan expuestos en la tabla 5 .

Para las dependientes: NOBIGDATA, en cuanto al Sexo, AMENAZA, en cuanto a Edad, NOREDES, en relación al Sector y NOMIEDO, en lo que respecta a Tamaño, 
sus márgenes inferiores y superiores, al no alcanzar la unidad, indican una reducción de la fuerza o posibilidad de las asociaciones que se están estudiando.

En la asociación de NODESPER con el Sexo la unidad no está contemplada en ese margen. Sin embargo, éste es relativamente amplio, de unos seis puntos. La variable tiene alta especificidad, pero baja sensibilidad: utilizarla para pronosticar la respuesta a la variable NODESPER acertaría el 100\% de los valores cuando se tratara de mujeres, pero el $0 \%$ si se trata de hombres. Por tanto, no es una buena variable predictora. Esto mismo ocurre con otras posibles asociaciones.

En el caso de la dependiente NOSEGURID y su potencial asociación al Sector, sus $\mathrm{r}$ y OR coinciden con las obtenidas en las primeras estimaciones. El margen entre los valores inferior y superior es, no obstante, realmente amplio. Su capacidad predictora, aun siendo la mayor de todo el panel que se analiza -un 79,7\% para los que pertenezcan a Educación, Automoción y Transporte, y un 60,0\% para los que no pertenezcan a éstos-, sigue siendo, en general, bajo, del 70,2\%.

Por último, en cuanto a la variable NOPRIVAC, se da prácticamente coincidencia de las medidas $r$ y OR con las halladas en el estudio de la Chi cuadrado y la RR respectivas, para sendas variables de control, Sector y Tamaño. Sus márgenes son los más reducidos, con menos de cinco puntos de diferencia. Sin embargo, sigue dándose bajas capacidades de predicción en ambos casos. ¿Cabría entonces plantearse una modelización de esta variable en relación a las de control Sector y Tamaño? La reducida predicción no hace prever un modelo teórico acertado. Aún con ello, y teniendo en cuenta los posibles fenómenos de interacción y confusión con las otras variables de perfil, se procede a su estudio, pues establecer una ecuación para la motivación permitirá a los gerentes intervenir cuando el nivel de la misma lo demande (Quijano y Navarro, 2012).

\subsection{Posible modelo por regresión logística para NOPRIVAC}

En principio, el modelo debería contener sólo dos variables de control, las de Sector y Tamaño. Tras analizar las posibles interacciones entre Sector y Sexo, o Sector y Edad, y entre Tamaño y Sexo, o Tamaño y Edad, ninguno de los casos presenta una clara evidencia de interacciones. Se valora el incluir en la ecuación, o modelo, a la variable Sexo, pues el término de interacción Tamaño*Sexo es el único que presenta significación estadística (0.019).

Una vez tratada la interacción, se procede a estudiar la posibilidad de confusión entre las variables de control. Tras valorar el conjunto de recomendaciones que dan los expertos (Aguayo \& Lora, 2007) para tomar una decisión al respecto (tabla 6) se concluye que la variable Edad produce confusión en la de Sector, por lo que se incluirá en el modelo a tratar. 
Tabla 6. Estudio de la posibilidad de confusión entre variables

\begin{tabular}{|l|l|l|l|l|}
\hline \multicolumn{1}{|c|}{ Condiciones } & \multicolumn{1}{|c|}{$\begin{array}{c}\text { Sector y } \\
\text { sexo }\end{array}$} & Sector y edad & $\begin{array}{l}\text { Tamaño y } \\
\text { sexo }\end{array}$ & $\begin{array}{l}\text { Tamaño y } \\
\text { edad }\end{array}$ \\
\hline OR & $\begin{array}{l}\text { Antes 2.685 } \\
\text { Ahora 2.744 }\end{array}$ & $\begin{array}{l}\text { Antes 2.685 } \\
\text { Ahora 4.044 }\end{array}$ & $\begin{array}{l}\text { Antes 2.374 } \\
\text { Ahora 2.353 }\end{array}$ & $\begin{array}{l}\text { Antes 2.685 } \\
\text { Ahora 2.967 }\end{array}$ \\
\hline Variación OR $>10 \%$ & NO & SÍ & NO & SÍ \\
\hline $\begin{array}{l}\text { No modificación sustancial } \\
\text { del intervalo de confianza }\end{array}$ & SÍ & $\begin{array}{l}\text { NO. Lo aumenta } \\
\text { notablemente: } \\
\text { >imprecisión }\end{array}$ & SÍ & $\begin{array}{l}\text { NO. } \\
\text { Lo aumenta. }\end{array}$ \\
\hline $\begin{array}{l}\text { No modificación en la significación } \\
\text { estadística de Wald }\end{array}$ & SÍ & Sí & Sí & Sí \\
\hline $\begin{array}{l}\text { Significación estadística de la } \\
\text { variable de confusión }\end{array}$ & NO & Sí (0.047) & NO & NO \\
\hline $\begin{array}{l}\text { El intervalo de confianza } \\
\text { no contiene el valor nulo }(=1)\end{array}$ & NO & $\begin{array}{l}\text { Sí } \\
\text { (por muy poco) }\end{array}$ & NO & NO \\
\hline Decisión & $\begin{array}{l}\text { Rechazar la } \\
\text { confusión }\end{array}$ & $\begin{array}{l}\text { Aceptar la } \\
\text { confusión }\end{array}$ & $\begin{array}{l}\text { Rechazar la } \\
\text { confusión }\end{array}$ & $\begin{array}{l}\text { Rechazar la } \\
\text { confusión }\end{array}$ \\
\hline
\end{tabular}

En la tabla 7 se muestran los principales indicadores que evalúan la bondad de un ajuste realizado por regresión logística. A pesar de que ninguna de las propuestas crea un modelo que cumpla la totalidad de los mismos, es el tercero, es decir, una vez que, a las variables de control de partida, Sector y Tamaño, se les añade la que interactúa con Tamaño (Sexo) y la que origina confusión con Sector (Edad), el que más se aproxima.

\section{Tabla 7. Indicadores de las variables del modelo}

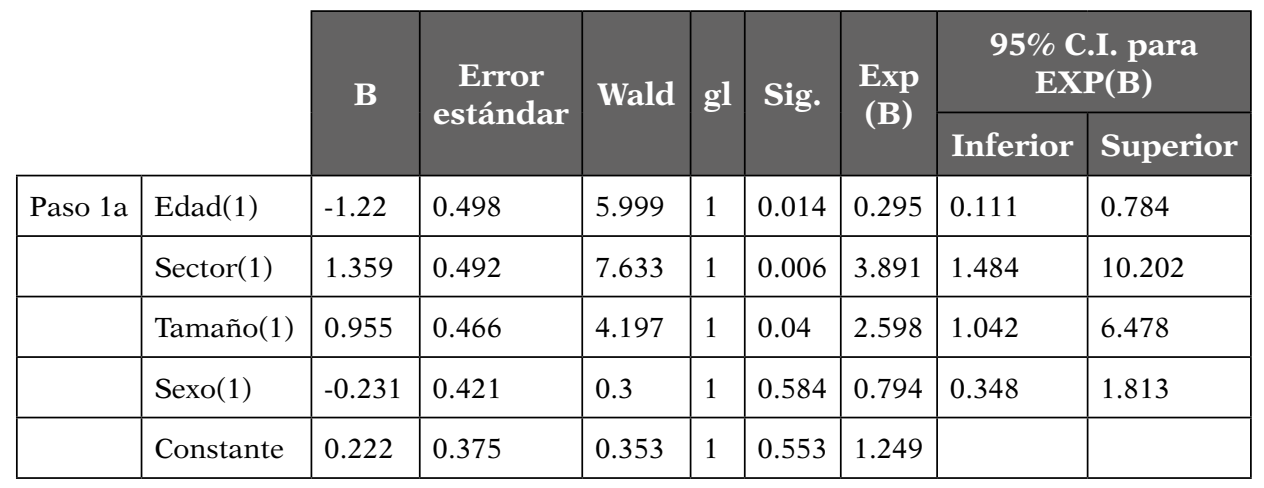

a. Variables especificadas en el paso 1: EDAD, SECTOR, TAMAÑO, SEXO.

Aunque la variable Sexo no presenta significación estadística, aun por muy poco, el resto sí, confirmando que explican a la variable dependiente NOPRIVAC. La mayor fuerza en esta relación la crea la variable Sector $(\operatorname{Exp}(B)=3.891$, la más alejada de la unidad, seguida de Tamaño $(\operatorname{Ex}(B)=2.598$, que fueron las variables señaladas desde el inicio de este análisis como de mayor capacidad predictora para NOPRIVAC. 
Observando el signo de B, el sentido de la explicación, o relación de causalidad, es que una persona no joven (de edad superior a 35 años) teme más esta cuestión que una persona joven; que las personas que no trabajan en los sectores de Educación y Automoción y transporte son las más temerosas en este sentido; y que las que lo hacen en las microempresas y PYME lo son más que las que lo hacen en las grandes empresas.

El modelo, no obstante, adolecería de falta de una bondad adecuada en su ajuste, dados los reducidos porcentajes de pronóstico acertado, por lo que se decide no construirlo.

\section{Conclusiones y discusión}

El estudio muestra una clara aceptación de los factores motivacionales propuestos, y una discrepancia mayor en cuanto a la valoración de los «temores digitales», especialmente cuando se categoriza por sectores económicos.

En cuanto a las motivaciones, no se han encontrado estudios que hayan incluido cuestiones similares a las aquí planteadas, localizadas en la era digital. No obstante, investigaciones de los últimos años, como la de Shannon (2017), confirman que la comunicación honesta y abierta entre compañeros, así como el reconocimiento de la labor bien hecha, son dos de los motivadores más potentes (en este caso, en el sector público australiano). Ambientado en la tecnología, Li et al. (2004) habían utilizado la Teoría de Herzberg para medir el desarrollo del servicio de tratamiento de datos, tanto sus posibilidades económicas, como la aceptación de su utilidad por el mercado, en sus distintas fases. No trataron, sin embargo, los factores motivacionales o los factores a higienizar para un trabajador actual, en la era digital. Mucho más recientemente, Mamedov et al. (2019) se refieren a la importancia que tienen, en el deseo o la motivación de empleados y empleadores rusos y azerbaiyanos de desarrollar su propio capital humano y el de la empresa, las diversas visiones o características de las distintas generaciones de compañeros que conviven en la misma: Generación X, Generación Y (millennials) y Generación Z, en el marco de la Cuarta Revolución Industrial. Como característica de los tiempos actuales, Jarupathirun y De Gennaro (2018) analizan el nivel de rotación en el empleo en Bangkok, Tailandia, y, a través, igualmente, de la Teoría de Herzberg, relacionan éste con aspectos en el trabajo. Así, concluyen que la relación con los compañeros, el reconocimiento, la seguridad en el trabajo y la remuneración son los factores que más influyen a la hora de optar por abandonar una empresa. Mucho menor poder, en este sentido, tienen cuestiones como el logro, la importancia del trabajo, el crecimiento, la política de la compañía y las relaciones con supervisores. Abolhoseini et al. (2018) llegan a la conclusión de que la calidad en el trabajo, la responsabilidad asumida, la categoría del mismo, así como la seguridad y el salario resultan los motivadores de mayor valor entre los trabajadores consultados (terapeutas de rehabilitación en sector sanitario de Teherán). También en este estudio se manifiesta una clara relación entre la variable sociodemográfica edad y el nivel de motivación laboral. Por su parte, Warrier et al. (2018) enmarcan su análisis en una actividad laboral propia de la era digital, el sector de las TI (Tecnologías de la Información) concretamente en la India, descubriendo que, en contra de lo que la propia Teoría de Herzberg sostiene, los factores higiénicos 
juegan un papel más importante en la predicción de la satisfacción laboral en esta muestra, cuestión atribuida a las diferencias culturales frente a Occidente. Pero, de nuevo, los estudios, incluso los actuales, toman los factores tradicionales de la Teoría de Herzberg; no aportan, como lo hace este trabajo, posibles nuevos factores propios de la implantación de lo digital. Escardibul y Afcha (2017) incluyen en su estudio nuevas variables, propias del entorno, social y laboral, de un doctor universitario, pero ninguno relativo a la era digital.

En el presente trabajo se concluye que se produce asociación entre determinadas variables de perfil sociodemográfico y varios de los temores tratados, especialmente fuerte entre las variables que miden el sector y el tamaño de la empresa en cuanto al miedo a perder privacidad ante la conexión digital a través de redes en el trabajo. Intentado un posible modelo para representar esta relación, se decide descartarlo por deficiente ajuste en el pronóstico de los valores.

El trabajo no está exento de limitaciones. Así, los ítems no tienen una base firme en la literatura, dada la novedad del tema, aunque se ha solventado con la intervención de expertos, por lo que supone una limitación relativa. La muestra, aun superando la centena, está compuesta por un número reducido de participantes. Sería oportuno trabajar con muestras mayores, así como en otros territorios y en distintos momentos a lo largo del tiempo, para estudiar el posible cambio de comportamiento ante las mismas cuestiones. Por otro lado, al «nominalizarse» las variables cuantitativas en dos categorías: «Teme», o «No teme» se es consciente de la pérdida de parte de la información, aunque es el camino adoptado para poder realizar un análisis de regresión logística.

Además de lo ya señalado sobre similares estudios con otras muestras poblacionales, se propone un tratamiento alternativo de la información recabada, cual es el de un análisis factorial exploratorio de las variables, que las agregue en constructos de mayor información, y un consiguiente análisis factorial confirmatorio posterior; y ello tanto para los factores motivacionales como para los que requieren medidas higiénicas. La dependencia o asociación se realizaría en último lugar, partiendo de las nuevas variables fruto de dichos constructos.

No obstante, siendo una primera aproximación, resulta novedoso el aportar factores característicos del entorno digital laboral, y, en concreto, desde el enfoque de la Teoría de la Motivación de Herzberg. Estudios como éste pueden ser útiles a todo tipo de gerentes, pues es de suponer que todo gerente busca lograr un ambiente sano y motivador y activaría medidas «higiénicas» para paliar esos temores. Pero, sobre todo, servirá a aquéllos que plantean su labor desde el modelo del «Happiness Management», bajo la convicción de que eliminar insatisfacciones es parte del camino hacia un mayor rendimiento del conjunto. 


\section{Referencias}

Abolhoseini, E., Mobaraki, H., Kamali, M., et al. (2018). Relationship Between Performance Evaluation and Therapists' Job Motivation of Rehabilitation Centers and Public Hospitals of Tehran Based on Herzbergs' Two-Factor Model. Archives of Rehabilitation, 18(4), 316-327. https://doi.org/10.21859/JREHAB.18.4.6

Aguayo, M., \& Lora, E. (2007). Cómo hacer una regresión logística binaria "paso a paso": análisis multivariante. Docuweb FABIS, Recuperado de https://bit.ly/2ZgksQ5 [Fecha de consulta: 14 de mayo de 2019].

Arslan, M., \& Roudaki, J. (2019). Examining the role of employee engagement in the relationship between organisational cynicism and employee performance. International Journal of Sociology and Social Policy, 39(1-2), 118-137. https://doi.org/10.1108/IJSSP-06-2018-0087

Baptiste, N.R. (2007). Tightening the link between employee well-being at work and performance. Management Decision, 46(2), 284-309. https://doi.org/10.1108/00251740810854168

Bashir, M., Saleem, A., \& Ahmed, F. (2019). Akhuwat: Measuring Success for a Non-profit Organization. Asian Journal of Management Cases, 16(1), 100-112. https://doi. org/10.1177\%2F0972820119825973

Cech, P., Beranek, M., \& Chromy, J. (2015). Job Satisfaction of Managers Working for Hotels And Hotel Chains in the Czech Republic. En: 26th International-Business-Information-Management-Association Conference, November, 11-12. Innovation Management and Sustainable Economic Competitive Advantage: from Regional Development to Global Growth Vols. I-V, pp. 668-676. Recuperado de https://bit.ly/2Wyc55F

Corbetta, P. (2010). Metodología y Técnicas de Investigación Social. Madrid, España: McGraw-Hill/Interamericana.

Cropanzano, R., \& Wright, T.A. (2001). When a "Happy" Worker Is Really a "Productive" Worker: A Review and Further Refinement of the Happy-Productive Worker Thesis. Psychology Journal Practice and Research, 53(3), 182-199. https://psycnet.apa.org/doi/10.1037/10614087.53.3.182

Emiroglu, O., Guneyli, A., \& Burgul, N. S. (2017). Motivational Sources of Teachers in a Developing Country. Revista de Cercetare si Interventie Sociala, 57, 51-66. https://search.proquest.com/ docview/1923729164? accountid=32861

Escardibul, J.-O., \& Afcha, S. (2017). Determinants of the job satisfaction of PhD holders: an analysis by gender, employment sector, and type of satisfaction in Spain. Higher Education, 74(5), 855-875. https://doi.org/10.1007/s10734-016-0081-1

Fisher, C. (2010) Happiness at work. International Journal of Management Review, 12(4), 384-412. https:// doi.org/10.1111/j.1468-2370.2009.00270.x

Gaitan, I., Breton, D. C., Urbano, H. L. C., Mahecha, C., \& Arteaga, M. (2015). Todo es cuestión de actitud. Gestión de la felicidad. Harvard Deusto Business Review, 6-17. Recuperado de https://bit.ly/2WsYqrJ [Fecha de consulta: 30 de mayo de 2019].

Ghahremani Germi, N., Ghahremani Germi, M., \& Delghavi, E. (2014). The effect of management factors and job satisfaction on human resources efficiency: A case study of paramedical employees of public hospitals in Ardabil. International Journal of Organizational Leadership, 1(1), 18-37. http://dx.doi.org/10.33844/mihs.2015.60247

González-Díaz, J., Ochoa-Dearco, E., \& Cardona-Arbeláez, D. (2018). Modelo conceptual de Gerencia de la Felicidad. Orbis. Revista Científica Electrónica de Ciencias Humanas, 17-32. Recuperado de https://bit.ly/2WsYxDA [Fecha de consulta: 20 de mayo de 2019].

Herzberg, F., B. Mausner, B. \& Snyderman, B. B. (1959). The motivation to work. New York: Wiley.

Hosie, P.; Sharma, P., \& Kingshott. R. P.J. (2019). Happy-performing managers thesis Testing the mediating role of job-related affective outcomes on the impact of role stressors on contextual performance. International Journal of Manpower, 40(2), 356-372. https://doi. org/10.1108/IJM-04-2018-0124 
Jarupathirun, S. \& De Gennaro, M. (2018). Factors of work satisfaction and their influence on employee turnover in Bangkok, Thailand. International Journal of Technology, 9(7), 1460-1468. https://doi.org/10.14716/ijtech.v9i7.1650

Kang, J. H., Matusik, J. G., Kim, T. Y., \& Phillips, J. M. (2016). Interactive effects of multiple organizational climates on employee innovative behavior in entrepreneurial firms: A cross-level investigation. Journal of Business Venturing, 31(6), 628-642. https://doi. org/10.1016/j.jbusvent.2016.08.002

Khoshnevis, H., \& Tahmasebi, A. (2016). The Motivation System in a Governmental Organization. En McKenna, B., Ardabili, F. S., y Faghih, N. (Eds.), 3rd International Conference on New Challenges in Management and Business: Organization and Leadership, 2 May 2016, (pp. 212-218). Dubai, UAE. https://doi.org/10.1016/j.sbspro.2016.09.027

Koenes, A. (1996). Gestión y motivación del personal. Madrid, España: Ediciones Díaz de Santos.

Li, R.P., Rong, R., \& Lu, T.J. (2004). Managing the relationship of technology and market in different stages of data service development. En 6th International Conference on Computer Communication (ICCC 2004), September 15-17. Beijing, Republic China.

López, J.P., \& Fierro, I. (2015). Determinantes de la felicidad en los administradores: una investigación realizada en las farmacias del Grupo Difare en Ecuador. Universidad \& Empresa, 17(29), 181-211. https://dx.doi.org/10.12804/rev.univ.empresa.29.2015.08

López, J. (2005). Motivación laboral y gestión de recursos humanos en la teoría de Frederick Herzberg. Rev Invest Fac Ciencias Administrativas UNMSM, Julio 2005, 25-36. Recuperado de https://bit.ly/2Km346j [Fecha de consulta: 30 de abril de 2019].

Mamedov, Z. F.; Mineva, O. K.\& Glinchevskiy, E. I. (2019). Innovative Approach to Human Capital Management under conditions of strong turbulence of Fourth Industrial Revolution. En Ibrahimov, M., Aleksic, A., y Dukic, D. (Eds.), 37th International Scientific Conference on Economic and Social Development- Socio Economic Problems of Sustainable Development, febrero 14-15, (pp. 1518-1525). Baku, Azerbaijan. Recuperado de https://bit.ly/2MFhqDC

Meyers, M.C., van Woerkon, M., \& Bakker, A.B. (2013). The added value of the positive: a literature review of positive psychology interventions in organizations. European Journal of Work and Organizational Psychology, 22(5), 618-632. https://doi.org/10.1080/1359432X.2012.694689

Miraz, M., Excell, P.S., \& Maaruf, A. (2016). User interface (UI) design issues for multilingual users: a case study. Universal Access in the Information Society, 15(3), 431-444. https://doi. org/10.1007/s10209-014-0397-5

Moccia, S. (2016). Felicidad en el trabajo. Papeles del Psicólogo, 37(2), 143-151. Recuperado de https://bit.ly/2R3Iond [Fecha de consulta: 30 de mayo de 2019].

Petersen, E., Wascher, M., \& Kier, K. (2017). Analysis of pharmacy student motivators and deterrents for professional organization involvement. Currents in Pharmacy Teaching and Learning, 9(4), 543-550. https://doi.org/10.1016/j.cptl.2017.03.024

Price, S., \& Reichert, C. (2017). The importance of continuing professional development to career satisfaction and patient care: meeting the needs of novice to mid-to late-career nurses throughout their career Span. Administrative Sciences, 7(2). https://doi.org/10.3390/admsci7020017

Quijano, S.D., \& Navarro, J. (2012). La autoeficacia y la motivación en el trabajo. Apunt. Psicol, 30(1-3), 337-349. Recuperado de https://bit.ly/2I5kWmA [Fecha de consulta: 15 de mayo de 2019].

Rahman, K.-U., Akhter, W., \& Khan, S.U. (2017). Factors affecting employee job satisfaction: A comparative study of conventional and Islamic insurance. Cogent Business $\mathcal{E}$ Management, 4(1). https://doi.org/10.1080/23311975.2016.1273082

Ravina, R., Villena, F., \& Gutiérrez, G. (2017). Una aproximación teórica para mejorar los resultados de innovación en las empresas desde la perspectiva del "Happiness Management. Retos. Revista de Ciencias de la Administración y Economía, 7(14), 113-129. http://dx.doi. org/10.17163/ret.n14.2017.06

Rizkallah, E. G., \& Seitz, V. (2017). Understanding student motivation: a key to retention in higher education. Scientific Annals of Economics and Business, 64(1), 45-57. https://doi.org/10.1515/ saeb-2017-0004 
Robbins, S., \& Judge, T. (2009). Comportamiento Organizacional. Decimotercera edición. México: Pearson Educación.

Romero-Rodríguez, L.M., Castillo-Abdul, B., \& Ravina, R. (2019). Comunicación para la efectividad laboral y el Happiness Management: revisión crítico-analítica de la literatura. Revista Mundo Indess, 1(1), 17-27. Recuperado de https://bit.ly/2EX2R8c [Fecha de consulta: 10 de mayo de 2019].

Shannon, E.A. (2017). Motivating the workforce: Beyond the 'two-factor' model. Journal of the Australian Healthcare $\mathcal{E}$ Hospitals Association, 43(1), 98-102. https://doi.org/10.1071/AH16279

Taipale, S., Selander, K., Anttila, T., \& Nätti, J. (2011). Work engagement in eight European countries: the role of job demands, autonomy, and social support". International Journal of Sociology and Social Policy, 31(7-8), 486-504. https://doi.org/10.1108/01443331111149905

Torkaman, F., Farhang, S., Zakerian, S.A., et al. (2017). A study on the effect of job burnout and stress on job satisfaction among teachers of exceptional schools. Pharmacophore, 8(5), 1824. Recuperado de https://bit.ly/2F2vErU [Fecha de consulta: 24 de abril de 2019].

Verma, P., \& Sharma, D. (2018). Quality of Work Life in Academics with reference to Motivational Theories. Pacific Business Review International, 11(4), 159-165. Recuperado de https://bit. ly/2K4DkOB [Fecha de consulta: 10 de mayo de 2019].

Warrier, A. G., \& Prasad, R. (2018). Motivators, Hygiene Factors and Job Satisfaction of Employees in IT Sector in India. En 7th International Conference on Computing, Communications and Informatics (ICACCI), September 19-22, pp. 477. Bangalore, India; IEEE. https://doi.org/10.1109/ ICACCI.2018.8554403

Zelenski, J.M., Murphy, S.A., \& Jenkins, D.A. (2008). The Happy-Productive Worker Thesis Revisited. Journal of Happiness Studies, 9(4), 521-537. https://doi.org/10.1007/s10902-008-9087-4 